\title{
Filosofizzazione del cristianesimo
}

\section{The Philosophization of Christianity}

\author{
ALDO MAGRIS \\ Università di Trieste \\ magris@units.it, ORCID: 0000-0002-4692-7931
}

\begin{abstract}
Riassunto: Laddove la cultura ellenica nei suoi aspetti più importanti scomparve all'avvento della religione cristiana, solo la filosofia greca sopravvisse, anzi trovò nella nuova situazione culturale forme di sviluppo originali. Perciò noi dovremmo parlare di filosofizzazione, piuttosto che di ellenizzazione del cristianesimo. Inizialmente gli autori cristiani erano avversi alla filosofia, ma poi prevalse la linea di Giustino, di Clemente e di Origine secondo cui essa poteva rappresentare una propedeutica a una comprensione colta della fede: certo non tutta la filosofia greca ma soltanto il filone platonico e aristotelico. Da queste specifiche fonti i Padri della Chiesa trassero concetti e argomentazioni utili per due scopi: il primo, la dimostrazione del libero arbitrio a supporto dell'etica, e della provvidenza divina in funzione della teodicea; il secondo, la critica dei miti e culti pagani e delle contemporanee sette eretiche. In particolare la confutazione del manicheismo impiegò su larga scale le strutture formali dell'ontologia aristotelica. La filosofizzazione divenne parte integrante dell'identità e della visione cristiana del mondo.
\end{abstract}

Parole chiave: Ellenizzazione del cristianesimo, Patristica, teodicea, ontologia aristotelica

Abstract: Whereas all major aspects of the Hellenic civilization faded away at the triumph of the Christian religion, only Greek philosophy remained, and found some original developments inside the new cultural environment. Therefore, we should speak of philosophization rather than the hellenization of Christianity. Many earlier Christian authors were indeed hostile towards philosophy, yet Justin's, Clement's and Origen's line prevailed, saying it could provide a propedeutic to the understanding of faith, although not as a whole but specifically in its Platonic and Aristotelian tradition. The Church Fathers gathered from the repertoire of these sources most notions and topics useful for two aims: firstly, the assumption of free will supporting ethics, and the demonstration of divine providence as a pivot for theodicy; secondly, the refutation of pagan idolatry and contemporary heresies. Above all, against the Manicheans they made wide use of the key concepts drawn from the Aristotelian ontology. The philosophization has become an integral part of Christian identity and vision.

Keywords: Hellenization of Christianity, Patristic Thought, Theodicy, Aristotelian ontology

\section{I primi cristiani e la filosofia greca}

Oltre un secolo fa lo storico della Chiesa Adolf von Harnack introdusse il concetto di «ellenizzazione del cristianesimo» (Hellenisierung des Christentums), concepita come una sorta di «patologia» che avrebbe infettato il vangelo di Gesù originariamente rivolto alla salvezza dei peccatori e allavvento del Regno di Dio provocandone la «mondanizzazione» (Verweltlichung); insorta in forma «acuta» ed estrema 
(akut) nello gnosticismo, essa si sarebbe poi diffusa in forma cronica o «progressiva» (allmählich) nella Patristica e nella Scolastica cattolica, e solo la Riforma luterana avrebbe avuto l'intenzione di sanarla, senza peraltro riuscirci del tutto ${ }^{1}$. La fortuna di questa celebre tesi credo dipenda dalla sua capacità di evocare l'ideale di un cristianesimo autentico la cui primitiva "purezza" bisognerebbe oggi recuperare, liberandolo da sovrastrutture culturali aliene o devianti rispetto alla sua finalità escatologica. Non per nulla fino a qualche decennio orsono c'erano ancora zelanti cristiani (protestanti o cattolici) che si davano da fare con un programma di "de-ellenizzazione", magari valorizzando le radici ebraiche e bibliche del messaggio evangelico. Purtroppo le cose non sono mai così semplici. Nessuna forma religiosa sfugge a una certa "mondanizzazione", perché nel momento in cui entra nella storia è giocoforza che si appoggi a determinati paradigmi culturali, e l'eventuale sostituzione di un paradigma con un altro (nel nostro caso, di un paradigma ellenistico al posto di uno giudaico-apocalittico) non è in sé una distorsione ma un processo evolutivo normale, anzi il più delle volte costruttivo, che inerisce come tale a tutti i fenomeni storici. Lungi dal deplorare che una "ellenizzazione" ci sia stata, nostro compito è piuttosto comprendere in quali termini reali si sia svolta ed a quali reali esigenze venisse incontro.

In generale, "ellenizzazione" vuol dire adozione della lingua e della cultura greca, vale a dire degli Héllènes come si chiamavano ancora in età imperiale, a prescindere dal paese di nascita e dall'etnia di appartenenza. Ma questo era nell'ordine delle cose dal momento che il cristianesimo, fallito il tentativo di predicare nelle sinagoghe (soprattutto dopo che i rabbini di indirizzo farisaico ne presero l'egemonia), doveva per forza rivolgersi per lo più fuori dall'ebraismo ad un uditorio già ellenizzato presso il quale raggiunse ben presto un considerevole successo. Il greco divenne così la lingua ufficiale della nuova religione (più tardi affiancato dal latino in Occidente) e in essa sono composti tutti i suoi documenti nei primi secoli, opera di persone che oltre a padroneggiarlo perfettamente erano di solito anche ben istruiti nella medesima paideia ellenistica dei "pagani". In realtà poi Harnack implicitamente precisa la sua ambigua parola dordine facendo capire che la Hellenisierung in pratica è consistita nell'influsso che la filosofia greca, anzi nello specifico la «filosofia della religione» platonica ha esercitato sulla formazione del dogma cristiano. Allora il problema cambia: non si è trattato affatto di una "ellenizzazione" (al cristianesimo sono del tutto estranei aspetti fondamentali della civiltà greca classica), semmai di una filosofizzazione. La tesi harnackiana si presenta quindi speculare a quella for-

1 Harnack, Lehrbuch der Dogmengeschichte I, 1; la tesi harnackiana è ribadita nella sua opera più divulgativa Das Wesen des Christentums. Il presente saggio è la versione modificata e aggiornata del $₫$ VII,5 del mio libro Destino, provvidenza, predestinazione (576-602). Su questo stesso argomento mi permetto di ricordare due altri miei lavori: "La filosofia greca e la formazione dell'identità cristiana" (2004) e "Platonismo e cristianesimo alla luce del Contro Celso", entrambi nella raccolta Itinerari della filosofia e delle religioni, $I$. 
mulata negli stessi anni da Nietzsche, che la religione cristiana non sarebbe se non "platonismo per il popolo» ${ }^{2}$. Ma perché il cristianesimo a un certo punto si è appropriato di una forma di pensiero così lontana dalla sua ispirazione? E in primo luogo, perché ciò è avvenuto in maniera così selettiva?

Dapprincipio i cristiani, peraltro ancora in buona parte ebrei, avevano ereditato dal giudaismo la viscerale avversione nei confronti della cultura grecoromana, anche qualora l'avessero studiata a scuola. Non solo ne contestavano i presupposti religiosi politeistici, ma trovavano abominevole la massa di rappresentazioni mitologiche della divinità che ne alimentava l'arte, la poesia, il teatro, i modelli della vita sociale. Secondo la visione comune ad ebrei e cristiani, l'idolatria in tutte le sue forme era stata introdotta nel mondo umano dagli angeli ribelli del Libro di Enoc (un mito contro altri miti!) e qualcuno faceva derivare dai loro perversi insegnamenti anche l'origine della filosofia, con la sua pretesa di insegnare all'uomo a «vivere bene» ${ }^{3} \mathrm{e}$ ad essere felice indipendentemente dalla Parola di $\mathrm{Dio}^{4}$. Dirà più tardi Agostino con analogo modo di pensare: «a che mi serve di vivere bene se poi non mi è dato di vivere sempre? $\|^{5}$. Persino Platone non sfuggiva al discredito, se riconosciamo una certa rappresentatività al colloquio (sia pure fittizio) avuto dall'ex-filosofo platonico Giustino con un vecchio cristiano al quale - a suo dire - doveva la propria conversione: questi ne attacca risoluto la credenza nell'immortalità dell'anima affermando che al pari di ogni altra creatura anche l'anima è soggetta alla morte, a meno che Dio non voglia conferirle lo «spirito» per la vita eterna! ${ }^{6}$ In ogni caso gli Apologisti criticano di frequente tutte le scuole filosofiche ${ }^{7}$.

Ben presto però l'atteggiamento del cristianesimo cambiò radicalmente e capofila del nuovo indirizzo fu proprio Giustino (il suo interlocutore l'aveva evidentemente convinto a farsi cristiano ma non a rinnegare la filosofia) con la sua teoria che la provvidenza divina ha sparso nel pensiero greco dei «semi» di razionalità e di verità (logoi spermatikói) per farli fruttificare appieno con l'avvento del Logos incarnato in Gesù Cristo ${ }^{8}$. Su questa strada lo segue Clemente alessandrino, che assegna alla filosofia nel mondo greco la stessa funzione espletata in quello ebraico

2 Nietzsche, Jenseits von Gut und Böse, Vorrede.

3 Cfr. SVF III, frr. 4, 16 e 17.

4 Cfr. Hermias, Gentilium philosophorum irrisio; Tertullianus, De anima 2; Clemens Alexandrinus, Strom. I, $16.80 ; \mathrm{v}, 1.10$ riferisce questa tesi senza condividerla, assieme a quella più estrema secondo cui la filosofia sarebbe un insegnamento del diavolo: ib. I, 1.18; 9.44; 16.80; 17.81; vi, 8.66; 17.159. Anche Justinus, 1 Apol. $2,5,14,54,68$ attribuisce ai «dèmoni» la degenerazione dell'umanità, ma non l'invenzione della filosofia.

5 Origenes, In Joh. xuv, 2.

6 Justinus, Dialogus 6.

7 Tatianus, Adversus Graecos 2, 3, 19, in questo caso contro i negatori dell'immortalità dell'anima; Teophilus, Autol. II, 2, contro i negatori della provvidenza; III, 5-6, contro gli eccessi di stoici e cinici; II 4, e III 7 , contro la teoria della reincarnazione; Clemens Alexandrinus, Strom. I, 11.52, contro il "materialismo" dei fisiologi. Anche gli gnostici valutavano negativamente le dottrine dei filosofi (salvo poi a sfruttarle a loro modo), cfr. Tractatus Tripartitus, NHC I, 109; Eugnostos, NHC III, 70; Cor. Herm., Asclepius 13.

8 Justinus, 1 Apol. 13, 21 e 46; 2 Apol. 10 e 13. 
dalla Legge mosaica, cioè di fornire ai due popoli una preparazione formativa (propaideia) tale da predisporli ad accogliere il messaggio cristiano9. Da seduzione diabolica e germe di corruzione la filosofia greca diventava in questa prospettiva teologico-storica uno strumento della salvezza, di cui solo il cristianesimo era in grado di portare a compimento la funzione "propedeutica". Ne derivò il fatto a prima vista sorprendente che proprio il cristianesimo ambì a presentare se stesso come una «vera filosofia» superiore a tutte le altre, ancorché una filosofia «barbara» in quanto avente le sue radici in una cultura diversa da quella ellenica ${ }^{10}$, ed evitò con cura fino almeno al V secolo di adottare il termine «teologia» con cui i filosofi platonici e stoici designavano la loro ermeneutica dei miti e dei culti tradizionali ${ }^{11}$. Si capisce, ciò comportava tacitamente una sostanziale alterazione di significato del termine. Un intellettuale cristiano può essere "filosofo" tanto poco quanto uno gnostico, poiché il suo pensare si svolge a partire da una rivelazione previamente accettata per atto di fede e condivisa dagli altri credenti, la quale non è liberamente interpretabile (qui a differenza degli gnostici) ma possiede già dei contenuti teorici propri e vincolanti in quanto trasmessi dalla «tradizione» della Chiesa (parádosis), disciplinati una «regola» (kanōn) che non può essere messa in discussione ${ }^{12}$. Gli Apologisti cristiani favorevoli alla filosofia si trovano così in una situazione contraddittoria, perché da un lato esortano a non limitarsi alla pura fede ma a svilupparla in una visione complessiva culturalmente ricca e razionalmente argomentata del reale (quella che Clemente chiama una "fisiologia' veramente gnostica») ${ }^{13}$; dall'altro ammoniscono che criterio della verità non è mai il sapere ma solo la fede, dono della grazia, istruita dalla viva voce del $\operatorname{Logos}$ fattosi uomo ${ }^{14}$. Dunque la filosofia non va esercitata per sé stessa ma

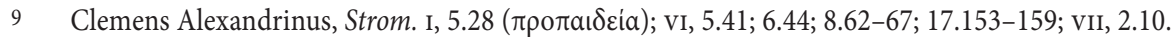

10 Cfr.Justinus, Dialoguscum Tryphone 8;ClemensAlexandrinus,Strom. I, 2.21;28.156;vI,7.54;mailcristianesimo non è filosofia per Tertullianus, De praescriptione haereticorum 7. L'espressione viene da Filone che designava l'ebraismo «filosofia mosaica» e «vera filosofia» (Decal. 96-98; Qu. Gen. II, 41) avvalendosi della nomea di "filosofi" degli ebrei (Theophrastus, De pietate fr. 13 [Pötscher]; Clearchus, fr. 6 [Wehrli]). La terminologia è variabile: l'antiellenico Tatianus, Adversus Graecos 31 e 35, contrappone alla philosophia la sophia della Rivelazione, mentre per Paolo (1Cor. 1.20) la sophia (= filosofia) dei Greci è «stoltezza». Bá $\rho \beta a \rho o \varsigma$

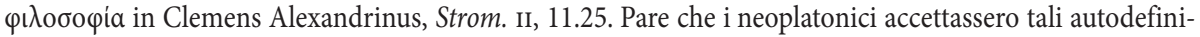
zioni: Alexander Lycopolitanus, Contra Manichaeos 1 parla di «filosofia» dei cristiani. La concordanza tra filosofia e cristianesimo si basava sullidea già del giudaismo alessandrino (Aristobulus fr. 3 [Holladay, 152]) che i pensatori greci sarebbero stati «ammaestrati dagli ebrei» o comunque influenzati dalla Bibbia (Clemens Alexandrinus, Strom. I, 1.10, cfr. Origenes, Contra Celsum v, 15; Justinus, 1 Apol. 59; lo gnostico Isidoro in Clemens Alexandrinus, Strom. vi, 6.53; Numenius, Fr. 8 [Des Places]).

11 Athenagoras (Ad Graecos 20) usa correttamente theología come sinonimo di "mitologia".

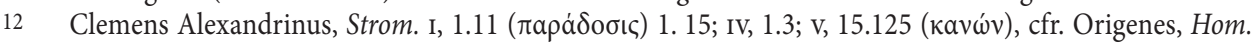
Jer. v, 141; In Joh. xxxII, 16.187. Elenco di contenuti dogmatici in Irenaeus, Haer. I, 10 e III-V; Hippolytus, Refut. x, 30-34.

13 Clemens Alexandrinus, Strom. Iv, 1.3. Per il significato di questo termine basato sulla distinzione clementina tra "falsa gnosi" (eretica) e "vera gnosi" (ortodossa) Rizzerio, Clemente di Alessandria, 93-98. 
solo per "coadiuvare" (syllambánesthai) la fede e fornirle un supporto ${ }^{15}$, o in altre parole non è la filosofia a porsi quale soggetto di un'indagine sulla religione (come facevano i filosofi greci), bensì la fede è il soggetto che valuta la filosofia e ne trasceglie quanto appaia utile ai fini della propria autocomprensione ${ }^{16}$.

Concepita in termini così essenzialmente strumentali, è chiaro che l'assimilazione del sapere filosofico offriva al cristianesimo notevoli vantaggi. Intanto permetteva all'intellettuale cristiano di fregiarsi della rinomanza e del prestigio che nel mondo antico circondava la figura del "filosofo", nonché di attingere senza conflitti di coscienza al patrimonio culturale della cosiddetta enkýklios paidéia, la formazione letteraria e filosofica impartita dalle scuole superiori in epoca ellenistica. Ma il fattore decisivo di questa scelta fu senza dubbio l’aver ravvisato una possibilità di convergenza su almeno tre punti basilari della propaganda cristiana: la critica della mitologia, la dottrina della provvidenza e la centralità dell'etica. Poiché essi sembravano trovare più integrale sostegno nella filosofia di Platone quale veniva insegnata dal contemporaneo medioplatonismo, non sorprende se ad essa fu riservata la maggior apertura di credito, al punto che Clemente afferma: «L’unica filosofia valida è quella di cui parla Socrate presso Platone» ${ }^{17}$.

\section{La critica della mitologia, il libero arbitrio e la provvidenza}

In realtà bisogna dire che la situazione nel cristianesimo dei primi secoli era molto più sfumata e che il pensiero filosofico (principalmente platonico, ma non solo) denota la sua presenza anche in autori dichiaratamente ostili alla filosofia in tutte le sue forme. La critica dei miti e dei culti pagani riprendeva anzitutto l'argomento già biblico, poi ripetuto a iosa dal giudaismo sia apocalittico sia rabbinico, sull'assurdità di adorare semplici manufatti costruiti dalla mano dell'uomo ${ }^{18}$; ma era unarma ormai spuntata perché era facile obiettare che nessuno venerava il marmo, il legno o il dipinto

15 Clemens Alexandrinus, Strom. I, 19.97; cfr. Origenes, Cels. III, 16; vI, 10; In Joh. xx, 17.288. Sulla funzione di supporto della filosofia: Justinus, 1 Apol. 10.4 e 53.12.

16 Clemens Alexandrinus, Strom. I, 11.53 citando 1Tess. 5.21: «esaminate tutto, tenete il buono». Cfr. Justinus, 2 Apol. 13: «I buoni principi da chiunque formulati appartengono a noi cristiani».

17 Clemens Alexandrinus, Strom. I, 19.92. Conformemente alla tendenza aristotelizzante del medioplatonismo, Clemente talvolta aggiunge alla «teologia di Platone» la «metafisica di Aristotele» (ib. I, 28.176). Gli stoici sono spesso apprezzati per l'etica (ib. II, 8.40; v, 9.58; Clemens Alexandrinus, Protr. 6.32), ma in generale lo stoicismo non poteva essere utilizzato ai fini di una fondazione teoretica della dottrina cristiana perché escludeva una trascendenza di Dio rispetto al mondo, ammessa invece sia da Platone (Timeo) sia da Aristotele (Metaph. XII); inoltre, la concezione stoica dell'eterno ritorno delle stesse cose era incompatibile con l'idea di creazione. Quasi tutti gli Apologisti sono di formazione medioplatonica; sembra invece che Origene abbia avuto maestri stoici.

18 Ad Diognetum 2; Justinus, 1 Apol. 9; Athenagoras, Ad Graecos 17; Theophilus, Autol. I, 1 e 10; II, 2; Clemens Alexandrinus, Protr. 4. 
in quanto tali bensì il significato da essi trasmesso ${ }^{19}$. Nei cristiani prevale allora un altro e più efficace argomento: l'ingenuità della rappresentazione antropomorfica e l'immoralità delle "favole" mitologiche con particolare riguardo ai comportamenti sessuali degli Dei. Qui si sente leco non solo della condanna platonica di Omero e di Eschilo nel secondo libro della Repubblica, ma anche del razionalismo illuministico che a partire da Senofane, Protagora, Prodico, fino a Carneade e Filodemo, attaccava la "superstizione" delle credenze religiose popolari. Stavano in questo filone, infatti, le premesse per la riduzione del mito a "favola" da demolire con un raziocinio condito di sarcasmo: il confronto fra gli esempi degli Apologisti e quelli carneadei riportati da Cicerone è a tale riguardo eloquente ${ }^{20}$. L'impiego di materiali filosofici da parte cristiana era dunque molto ampio e libero, per non dire spregiudicato, benché sempre guidato da un interesse. Anche chi apprezzava la "teologia" di Platone non si faceva scrupolo a rifornirsi di argomenti presso i razionalisti che nel denunciare le fandonie della religione contraddicevano precisamente al tentativo platonico e stoico di salvarla mediante un'interpretazione simbolica; salvo poi ad accusare di malevola ignoranza qualora analoghe accuse fossero state rivolte contro il cristianesimo. Del resto da parte pagana si procedeva allo stesso modo. Celso, l'autore di un energico pamphlet anticristiano di cui abbiamo notizia dal Contro Celso di Origene (seconda metà del II sec.), adotta contro la nuova religione uno stile argomentativo di pretta impronta illuministico-carneadea, pur essendo un platonico ben disposto a riconoscere la verità profonda delle religioni tradizionali.

$\mathrm{Ma}$ al di là dell'impiego selettivo delle fonti per ragioni polemiche, il vero nodo della questione da cogliere è il doppio inseparabile aspetto della filosofizzazione del cristianesimo, la quale ha un lato platonico nella sua elaborazione di un assetto culturale elevato alla dottrina cristiana ed un lato illuministico nel suo atteggiamento nei confronti delle religioni rivali, anzitutto del paganesimo ma poi anche in seguito. Eppure, non si tratta davvero di reale antitesi. Infatti proprio Platone, l'avversario dei sofisti e l'inquisitore dei miscredenti, palesa un fondo "illuministico" del suo pensiero nella misura in cui la religione per lui conta soprattutto per la sua funzione sociale e politica, poiché gli Dei (da lui identificati con gli astri, non con le figure della mitologia e del culto popolare) devono controllare il comportamento morale delle persone e garantire una sanzione ultraterrena per i cittadini che trasgrediscono le leggi

19 Cfr. Plutarchus, De Iside et Osiride 71, Celso in Origenes, Cels. III, 19, Corp. Herm., Asclepius 26.

20 Antropomorfismo: Athenagoras, Ad Graecos 18-21, 30; Theophilus, Autol. II, 3; Clemens Alexandrinus, Strom. v, 12.80; cfr. Cicero, De natura deorum I, 29-33; III, 12.29-13.32. Immoralità: Justinus, 1 Apol. 21, 25 e 54; Tatianus, Adversus Graecos 8 e 21; Theophilus, Autol. I, 9-10; II, 7-8; Athenagoras, Ad Graecos 21; Clemens Alexandrinus, Strom. viI, 4; Origenes, Cels. Iv, 48; cfr. Cicero, ib. III, 5.2; 6.15; 24.62. Confusione nel mondo divino: Athenagoras, Ad Graecos 14; cfr. Cicero, ib. III, 16.23. Altri argomenti di stile illuministico potevano essere ispirati dalle sentenze di critica religiosa assai frequenti in Euripide e altri drammaturghi del V-IV secolo, cfr. Theophilus, Autol. III, 7; Clemens Alexandrinus, Strom. v, 15.112; VII, 4.23; 6.30 . 
mettendo a rischio la solidità dello Stato. I due lati sono dunque due aspetti della stessa cosa. La filosofizzazione del cristianesimo è stata selettiva non perché ha preferito Platone ad altri filosofi, ma perché nel vasto orizzonte della civiltà greca ha ritenuto estranea ai suoi interessi l'esperienza del pensiero tragico, la riflessione sullapparire dei "fisiologi", la dialettica stoica di destino e libertà, e si è orientata invece verso il mondo dell'illuminismo che naturalmente è già un mondo complesso, in cui convivono condividendo certi presupposti (razionalità, autonomia dell'uomo, tecnica) sia l'istanza critica di Protagora o Carneade sia l'istanza metafisica di Platone e Aristotele.

Tuttavia per comprendere meglio quanto accaduto occorre allargare ulteriormente il panorama tenendo conto che il cristianesimo aveva uno sguardo bifronte: da una parte verso la grecità, dall'altra verso l'ebraismo. Anche qui fu fatta una selezione. I teorici della Chiesa hanno scartato le suggestioni dell'apocalittica (che pure godeva di un certo credito nel gruppo di Gesù) e le speculazioni radicali di Giobbe e di Qumran, mentre hanno scelto come interlocutore preferenziale il giudaismo farisaicorabbinico: non senza divergenze e conflitti, ovviamente, ma in quanto esso era l'espressione più coerente della Teologia del Patto considerata il cuore della visione biblica. Ora, dal nostro punto di vista il fariseismo in ambito ebraico è un fenomeno analogo ciò che è stato l'illuminismo in ambito greco, quanto meno per essere un movimento "laico" che dà il massimo rilievo al raziocinio nell'interpretazione dei precetti della Legge, all'intelligenza pratica dell'uomo, al suo "potere" (rəšūut $)^{21}$ di guadagnarsi un posto nel "mondo a venire" in virtù delle opere buone saggiamente scelte e liberamente compiute. Sotto questo profilo potremmo dire che la filosofizzazione del cristianesimo nasce dalla fusione di una duplice eredità, quella dell'illuminismo greco e quella dell'illuminismo ebraico. Ciò spiega chessa abbia sempre uno sfondo e una tendenza illuministica, nel senso più ampio del termine, non solo per quanto concerne la critica della mitologia, ma anche negli altri due punti di convergenza con la filosofia greca.

(1) Per la nozione di provvidenza occorre tenere sempre presente il significato razionalistico, non religioso, della parola pronoia (cioè programmazione, premeditazione) e la sua origine nel pensiero di Anassagora come applicazione sul piano cosmologico del pensiero della téchnē, l'operatività pianificata dell'intelligenza umana produttrice di ordine e di bellezza ${ }^{22}$. Nell'Antico Testamento essa è poco presente, per quanto strano possa sembrare, giacché nel creato si rileva non tanto la bellezza quanto l'esibizione di potenza del Creatore, e l'azione divina si manifesta nella storia piuttosto che nell'ordine della natura. Soltanto Filone riprende copiosamente le tematiche del provvidenzialismo filosofico con tutto il repertorio della teodicea, e con questo metodo procedono anche i Padri della Chiesa. Clemente motivava la sua venerazione per Platone col fatto che la sua filosofia indica un percorso di "risalita"

21 Il termine è attribuito a R. Aqiba nel Talmud (b Aboth 3).

22 Fondamentale su questo Theiler, Zur Geschichte [2 ed.]. 
(anábasis) dal sensibile allintelligibile, cioè dal mondo a Dio, e ciò avviene principalmente quando riconosciamo nellordine e nella bellezza del mondo la suprema intelligenza della Mente che lo ha prodotto, unico Principio dell'universo degno d'essere adorato ${ }^{23}$. Questa "prova teleologica" dell'esistenza di Dio, introdotta nel V sec. a.C. da Diogene di Apollonia, ritorna negli autori cristiani accompagnata dalla sua metafora tecnica. Basilio di Cesarea, ad esempio, definisce il cosmo un "manufatto" opera di una tecnica divina ben superiore a quella umana, proprio come Diogene ${ }^{24}$.

(2) La morale ha senza dubbio un ruolo di primo piano nel discorso degli Apologisti, per i quali il cristianesimo era l'unico rimedio al degrado della società grecoromana rappresentata come un mondo d'ingiustizia fatto solo di orge, bordelli e pederasti. Il cristiano doveva essere anzitutto un modello di integrità, un esempio di vita virtuosa ${ }^{25}$, e in tal profilo la vera realizzazione degli ideali già proclamati dalla filosofia. Ma la prassi morale si fonda sulla libera scelta e la conseguente responsabilità del soggetto per i suoi atti, dei quali riceve premio o ricompensa di fronte al giudizio umano o divino: qui il pensiero etico dell'illuminismo greco e il moralismo farisaico coincidevano in pieno, sicché nei Padri ritroviamo un miscuglio di argomenti presi da entrambe le fonti. Altrettanto frequente è perciò la citazione dal Deuteronomio: "Pongo davanti a te la vita e la morte», e quella dalla Repubblica: «la colpa è di chi ha scelto, Dio non è responsabile» ${ }^{26}$; il riferimento alla tesi aristotelica che l'uomo è principio dei suoi movimenti, e quello all'esegesi rabbinica di Genesi 1 secondo cui la "somiglianza" dell'uomo con Dio consiste nel suo esser "libero" e "autonomo" nelle proprie scelte ${ }^{27}$. Questa capacità di "autogestione" (autexousía) di cui l'uomo è stato dotato per natura viene nuovamente definito con il concetto aristotelico della potenza dei contrari ${ }^{28}$ sicché "sta-in-noi" optare per uno dei due esiti possibili operando una "scelta di propria iniziativa" (proáiresis hekoúsios) basata su una deliberazione razionale con ciò assumendoci la responsabilità

23 Clemens Alexandrinus, Strom. IV, 28.148; v, 6.2; 14.133; analoghe considerazioni in Athenagoras, Ad Graecos 16 e nell'antiplatonico Theophilus, Autol. I, 5. Per i cristiani la pronoia si occupa sia del tutto sia delle parti (Strom. viI, 6.9; 9.4). Sull'unicità del Principio (monoteismo) che i filosofi greci avrebbero solo in parte intravvisto: Athenagoras, Ad Graecos 5-7; Clemens Alexandrinus, Strom. I, 28.156; v, 14.102 e 133.

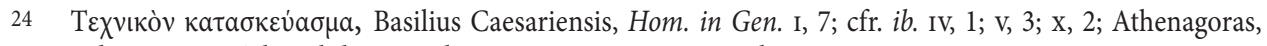
Ad Graecos 15; Theophilus, Autol. I, 5; Nemesius, De natura hominis 41.

25 Justinus, 1 Apol. 6 e 10; Teoph. I, 2; III, 9-15; Irenaeus, Haer. Iv, 36.6; 41.2; Origenes, Or. 20.

26 Citazione di Dt., 30.19: Ep. Apostolorum, 39, Ep. Barnabae, 18, e Hermas, Pastor Prec.vi, 1; Theophilus, Autol. II, 27; Irenaeus, Haer. IV, 39.1. Citazione di Resp. x, 15, 617e: Clemens Alexandrinus, Strom. I, 1.4; II, 16.75; IV, 23.150; v, 14.136; Paed. I, 69. Citazione abbinata di entrambi: Justinus, 1 Apol. 44.

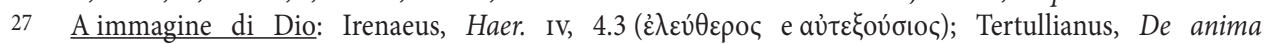

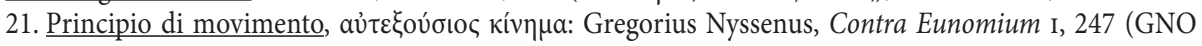
Online 1); Oratio catechetica 5 (GNO Online 16); cfr. Aristoteles, Eth. nic. III, 1, 1100a15. La somiglianza con Dio come possesso del libero arbitrio è anche esegesi rabbinica: Ber. Rabbà 21.5; Midrash a Cant. Cant. 1.9; Midr. a Esodo xxxis sul v. 23.10; Midr. a Numeri xvi su 14.19 (Wünsche). Cfr. Jervell, Imago Dei, 88-89, e Desalvo, L'oltre" nel presente, 117-121. 
delle conseguenze, cioè degli eventuali premi o castighi ${ }^{29}$. Su queste premesse è logico che per i Padri della Chiesa l'idea di destino non abbia nessun significato ${ }^{30}$. Una connessione fatale delle cose basata sul principio di causalità è inammissibile perché se il comportamento fosse l'effetto di cause esterne l'uomo - l'aveva detto Carneade sarebbe come una marionetta che si muove tirata da corde ${ }^{31}$. Il fatalismo astrologico viene pure confutato con i classici argomenti carneadei, per confermare la tesi paolina che l'avvento del Salvatore ha liberato il genere umano dalla servitù sotto le potenze cosmiche ${ }^{32}$.

\section{Confutazione della "mitologia" gnostica}

Ma l'appropriazione della filosofia greca da parte dei Padri della Chiesa, oltre che per uso interno, serviva anche e soprattutto quale supporto metodologico per affrontare il dibattito con le dottrine devianti dalla "Regola" ecclesiastica. Clemente alessan-

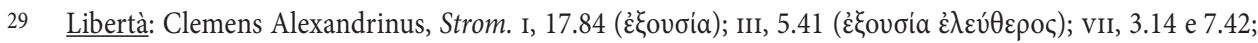

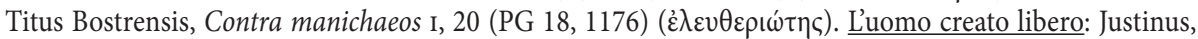

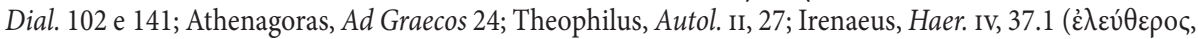

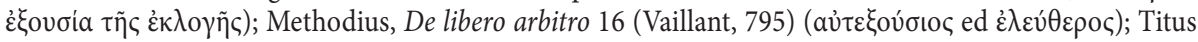
Bostrensis, Contra manichaeos II, 4 (PG 18, 1141a); Gregorius Nyssenus, De mortuis (GNO Online 34)

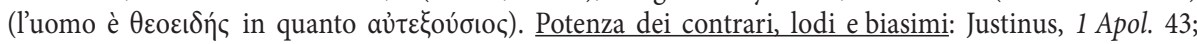
Justinus, 2 Apol., 7 e 11 (con apologo di Eracle al bivio); Theophilus, Autol. II, 24 e 26; Irenaeus, Haer.

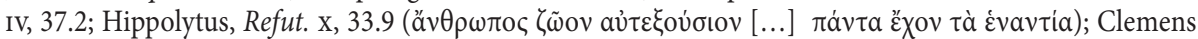

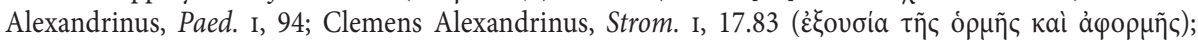
IV, 24.153; Origenes, Princ. I, 2.4 (possibilitas utriusque partis); III, 1.3; Methodius, De libero arbitro

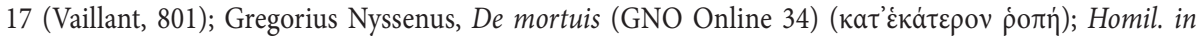
Ecclesiasten II (GNO Online 23); Homil. De beatit. 5 (PG 44, 1256); Basilius Caesareensis, Hom. in Gen. II, 15; Nemesius, De natura hominis 41 (Morani, 120); Titus Bostrensis, Contra manichaeos I, 27 (PG 18,

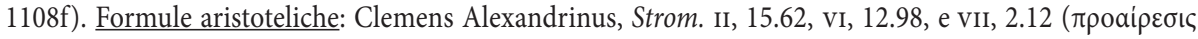

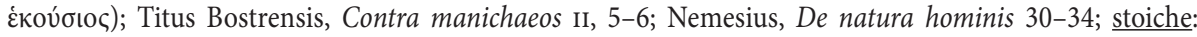

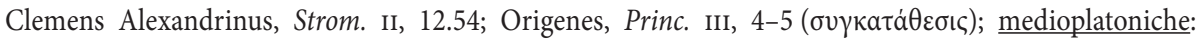
Justinus, 1 Apol. 43; Clemens Alexandrinus, Protr. 9.3; Nemesius, De natura hominis 38. La virtù stain-noi, il vizio può esser istigato ma non causato da dèmoni: Justinus, 2 Apol. 7; Clemens Alexandrinus, Strom. II, 12.107; vI, 12.98; Origenes, Princ. III, 2.2. Anche la fede è una «scelta» e un «assenso» libero: Clemens Alexandrinus, Strom. II, 2.8; 3.11; 6.27; v, 1.3; Origenes, Cels. III, 9.

30 "La heimarménē ole Moire non hanno niente a che vedere con Dio», Clemens Alexandrinus, Protr. 10.102. Cfr. l'analoga opinione di Anassagora: «moira è un nome vuoto» (Diels-Kranz, fr. A 66).

31 Clemens Alexandrinus, Strom. II, 3.11: «Se siamo tirati da forze naturali come da funi, come oggetti inanimati, niente esiste di nostra iniziativa o meno». Sul tema carneadeo dell'uomo "marionetta" del destino Schröder, «Marionetten».

32 Gal., 4.3 e 9; Ef., 6.12. Inizialmente gli Apologisti attaccavano l'astrologia solo in quanto opera dei dèmoni (Tatianus, Adversus Graecos 8-11); poi si usano gli argomenti carneadei: Origene nell'antologia intitolata Philocalia 23.14-18; ps. Clemens Romanus, Recognitiones Ix, 17; Diodorus Tarsensis, Contra fatum; Gregorius Nyssenus, Contra fatum (GNO Online 12); Basilius Caesariensis., Hom. in Gen. vi, 5-8; Eznik di Kolb, Su Dio 212-230. 
drino ripete spesso che il cristiano dev'essere in grado di fornire una "dimostrazione" razionale delle fede (apódeixis) per difendersi dalle insidie dei "sofisti" 3 . L'esigenza della dimostrazione era sorta in realtà proprio nellambiente dei sofisti del $\mathrm{V}$ secolo a.C., ma era stata recepita da Platone con finalità antisofistiche (benché sui medesimi presupposti illuministici) quando ad esempio "dimostra" l'immortalità dell'anima nel Fedone o la provvidenza divina nel decimo libro delle Leggi, e da Aristotele fissata in termini epistemologici negli Analitici, stavolta per arginare la sovversione della logica operata da quella nuova generazione di sofisti che erano i Megarici. In seguito la logica e la teoria della dimostrazione aristoteliche furono ampiamente adottate dai manuali medioplatonici sui quali si erano formati gran parte degli intellettuali cristiani: lo stesso Clemente ne fa un riassunto molto scolastico nel libro ottavo e ultimo dei suoi Stromati in cui si espongono i fondamenti del metodo argomentati$\mathrm{vo}^{34}$. Lorientamento platonico-aristotelico della filosofizzazione del cristianesimo si manifesta qui nel suo aspetto controversistico, perché i Padri ritrovano proprio nella lotta di Platone contro i sofisti e di Aristotele contro i Megarici il modello ideale del loro impegno per confutare la "falsa gnosi" degli eretici, ed è curioso che, mentre da un lato si accusavano gli gnostici dessere succubi dei filosofi pagani ${ }^{35}$, dall'altro solo in questi ultimi si ritrovavano i più efficaci antidoti contro il diabolico veleno dell'eresia. In pratica, però, l'esercizio della "dimostrazione" in tutta la Patristica è molto lontano dal metodo scientifico delineato da Aristotele. Come dice Clemente, «è dalla fede che noi desumiamo un convincimento apodittico»" sicché il più delle volte le asserzioni vengono "dimostrate" con argomenti di valore retorico più che epistemologico, per esempio il compimento delle profezie bibliche in Cristo o i valori morali corredati da passi scritturistici. Ma una sufficiente garanzia di validità era vista altresì nel selezionare argomenti dal secolare e ben documentato dibattito fra le scuole filosofiche, facendone uso a seconda della convenienza.

Contro gli gnostici vengono perciò impiegati tutti e tre i capisaldi della "filosofia" cristiana, a cominciare dalla critica della mitologia, che punta sulle incongruenze di una visione del divino esposta in termini narrativi e antropomorfici. È pura fantasia attribuire agli eoni l'“ignoranza" o la "passione", la capacità di produrre "ombra" mentre sono esseri incorporei, o quella di generare trattandosi di esseri spirituali ${ }^{37}$. Del resto questi eoni non sono che proprietà divine personificate, cioè - secondo la lo-

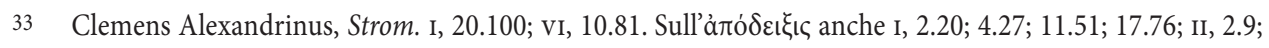
6.31; 9.43; VII, 7.20.

34 Sul medioplatonismo come tramite fra la logica aristotelica e il cristianesimo Lilla, Clement of Alexandria, 122.

35 Irenaeus, Haer. II, 14.2 e 33.2; Hippolytus, Refut. vi, 21; vir 14.29; IX, 7; Tertullianus, Praescr. 7.

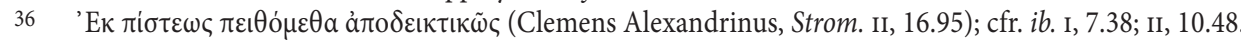

37 Irenaeus, Haer. II, 8.2; 17.10 ('lignoranza degli eoni circa il Padre era dottrina valentiniana cfr. Irenaeus, Haer. I, 2.1; Evangelium veritatis, NHC I, 24; sull'ombra dovuta all'ignoranza Tractatus Tripartitus, NHC I, 76). Sofia genera senza il suo compagno, «come le galline senza galli» (II, 12.4). Contro l'antropomorfismo gnostico anche Clemens Alexandrinus, Strom. II, 15.3; 17.2, 19.2; 42.2; IV, 11.2. 
gica categoriale aristotelica - dei "predicati", non dei "soggetti", che di conseguenza esistono alla stregua di meri "accidenti", non di entità sostanziali ${ }^{38}$. Spiegare poi l'origine del male con un guasto accaduto nel Pleroma è assurdo, perché non si capisce né come il Padre sia stato incapace di impedirlo né come potrebbe porvi rimedio essendone egli stesso vittima quasi per una sorta di fatalità. Molti eresiologi sottolineano tale contraddizione nell'idea gnostica di Dio ricorrendo al vecchio argomento epicureo: se Dio voleva evitare il male ma non poté farlo è impotente, se poteva ma non volle è malvagio ${ }^{39}$. Bisogna dunque concepire la divinità come del tutto aliena da passioni, da vicende romanzesche e da "miti tragici", cioè come puro "Intelletto" (nous) che ha creato e governa il mondo nella maniera migliore possibile ${ }^{40}$. Qui entrava in gioco il secondo caposaldo, la dottrina della pronoia, Gli gnostici di regola usavano questo termine per designare la prescienza divina, ma nella stragrande maggioranza dei casi erano decisamente avversi al pensiero provvidenzialistico, perché il mondo fabbricato e gestito dagli arconti inferiori è talmente pieno di difetti da manifestare non già la loro "provvidenza" (pro-noia) bensì la loro "de-menza" (apo-noia) ${ }^{41}$. Per i Padri, al contrario, in questo mondo tutto va talmente bene che il suo unico Creatore non può essere se non un Dio dotato della massima bontà e intelligenza; e a dimostrazione di ciò esibiscono il collaudato repertorio di argomenti diogeniano-platonici sulla perfetta regolarità dei moti celesti, la finalità dei processi naturali e la funzionalità degli organi del corpo umano ${ }^{42}$.

Il terzo caposaldo, l'etica, è rivolto contro il pensiero gnostico di una salvezza "per natura". Anche questo è considerato pura fantasia peggiore di quelle del paganesimo: una «mitologia delle diverse nature» la definisce Origene ${ }^{43}$. La tesi ben più "razionale" ripetuta continuamente dagli eresiologi è invece che tutti gli uomini sono in partenza uguali e non esistono individui già per loro natura predestinati a salvarsi o a perdersi, ma l'uno o l'altro esito dipende unicamente dalle scelte e dalla condotta di vita della singola persona. L'esempio è quello di Adamo, che Clemente ricor-

38 Irenaeus, Haer. II, 13.1-2 e 18.2-4.

39 Irenaeus, Haer. II, 3.2, 4.2. L'argomento epicureo ha varie testimonianze: Epicurea fr. 374 Usener = Lactantius, De ira dei IV, 13.19; anonimo in Nemesius, De natura hominis 43 (Morani, 130); Irenaeus, Haer. II, 5.3; cfr. anche ps. Clemens Romanus, Recognitiones II, 54, dove è argomento dello "gnostico" Simone contro Pietro.

40 Irenaeus, Haer. II, 13.3; Origenes, Princ. I, 1. Sul "mito tragico" (Hippolytus, Refut. vi, 42.2, che allude a Basilide) si i ricordi che l’agg. траүıкó in gr. classico voleva dire soltanto "teatrale" nel senso di magniloquente, esagerato, pomposo, ridicolo.

41 In copto APONOIA denomina la compagna di Jaldabaoth in Apocryphon Johannis, NHC II, 10, evidente gioco

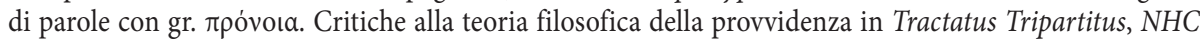
I, 101 (dove poi compare PRoNoIA nel senso di “prescienza"); Eugnostos, NHC III, 71. Solo i valentiniani ammettono una relativa "provvidenza" del Demiurgo (Tolomeo in Epiphanius, Panarion 33.3).

42 Irenaeus, Haer. II, 1-3; 9; III, 2.4; IV, 11.2; v, 2.1; 4.1; 17.1; Clemens Alexandrinus, Protr. 68-69; Clemens Alexandrinus, Paed. I, 62 e 88; Clemens Alexandrinus, Strom. Iv, 13.92; 26.165-167; vi, 1.6; viI, 2.8; 4.22; Origenes, Cels. v, 24; vi, 44.

43 Origenes, In Joh. xx, 33.287, con riferimento particolare ai valentiniani (Eracleone). 
da di aver portato durante un contraddittorio con un anonimo gnostico ${ }^{44}$ : egli era bensì perfetto, ma solo potenzialmente, ed ecco perché poi si è perso non avendo fatto la scelta adeguata a sviluppare tale sua potenzialità. Non è dunque la natura originaria di Adamo quello che conta, ma l'uso che concretamente ne ha fatto, poiché tanto la virtù quanto il peccato dell'uomo dipendono dall'“agire" (enérgeia), non dall'"essere" (ousia) ${ }^{45}$, e proprio in quanto atti responsabili vanno incontro alle dovute conseguenze. Dello stesso tenore unobiezione di Ireneo e di Origene contro l'antropologia "genetica" degli gnostici: i malvagi non sono tali perché "figli del diavolo" ma lo diventano dal momento in cui compiono le opere del diavolo, benché ciò nonostante persino i cosiddetti "figli del diavolo" possano diventare "figli di Dio" se si convertono ed abbandonano le "concupiscenze della carne» ${ }^{46}$. Su questo punto la posizione patristica coincideva con quella farisaica - dove l'uomo si salva mediante la prassi morale e si rovina a causa del peccato ${ }^{47}$ - e perciò coerentemente i Padri rivalutano l'importanza della Legge che a prescindere dai precetti rituali israelitici rimane secondo loro un presidio insostituibile della moralità comune $e^{48}$ : non a caso chi la contesta - lo gnostico - e quindi non riconosce vincoli etici di sorta finisce poi per «abbandonarsi alla libidine» ${ }^{49}$. Beninteso, ciò che salva non è la Legge ma la fede in Cristo; però anche la fede si basa sulla libera scelta e si manifesta come prassi ${ }^{50}$, per cui rientra essa stessa nella sfera della morale, con la sola distinzione desser accompagnata dalla giusta credenza religiosa. Comune ai rabbini è altresì l'argomento che Dio ha reso l'uomo non incapace di peccare, e ha consentito così quel margine di indeterminatezza costituito dal libero arbitrio, affinché la sua creatura fosse un essere ragionevole in grado di progredire e di acquistare dei meriti: altrimenti la salvezza sarebbe un processo automatico senza significato, senza pregio, e la perdizione un oscuro verdetto emesso in assenza di qualsiasi imputabilità di colpa ${ }^{51}$.

Il fariseismo faceva tali affermazioni nella convinzione, in un certo senso "illuministica”, che la soteriologia non può essere un mistero ma deve risultare com-

44 Clemens Alexandrinus, Strom. vi, 12.96.

45 Clemens Alexandrinus, Strom. IV, 13.93. Nel linguaggio aristotelico i due concetti non andrebbero contrapposti perché enérgeia è la stessa ousia in quanto posta "in atto" (cioè ẻv êp $p \omega \omega$, "in-opera"); i cristiani invece (v. infra, n. 84) intendono l'"atto" come azione soggettiva e non come realizzazione oggettiva preludendo così alluso moderno di "energia" nel senso di forza, capacità di agire.

Irenaeus, Haer. Iv, 41 (figli del diavolo); Clemens Alexandrinus, Paed. I, 29-31 (concupiscenza). Origenes, In Joh. xx, 5.38; 13.106 (anche i figli del diavolo possono diventare figli di Dio).

47 4Esdra 7.70; 8.55; 2Baruc 15; Psalmi Salom. 9.4 (Charlesworth, Pseudoepigrapha).

48 Irenaeus, Haer. Iv, 14-16; Clemens Alexandrinus, Protr. 108; Clemens Alexandrinus, Strom. I, 26.167; II, 7.32; 18.86; Origenes, In ep. Rom. v, 1 (PG 14).

49 Clemens Alexandrinus, Strom. III, 4.30.

50 Cfr. Clemens Alexandrinus criticando Basilide (Strom. v, 1.3) e Valentino (Iv, 13.89).

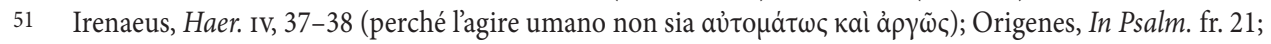
Hom. Num. x, 1; In ep. Rom. Iv, 12; De princ. I, 8.3; ps. Clemens Romanus, Recognitiones IX, 4; Methodius, De libero arbitro 16 (Vaillant, 799); 21 (Vaillant, 821); Gregorius Nyssenus, Oratio catechetica 31 (GNO Online 16); Titus Bostrensis, Contra manichaeos II, 3-4. Cfr. Talmud b, Chagiga 1. 
prensibile, ragionevole, per il credente e non cè modo migliore di farlo se non basandola sul soggetto, quindi sull'agire umano. Condividendo la medesima esigenza, i Padri la motivano riprendendo dalla loro cultura il tema chiave dell'illuminismo greco che, rispetto alla technē, la physis si capovolge in tychē perché quanto avviene semplicemente ad opera della natura va da sé, è scontato, è irrilevante, è "casuale", mentre un criterio di valore si può applicare solo nell'ambito delle iniziative prese e realizzate dall'uomo. Questo schema concettuale emerge con tutta evidenza nell'osservazione di Origene che la virtù non avrebbe alcun significato se non presupponesse deliberazione e sforzo nel conseguirla, e rispettivamente che i cosiddetti "figli del diavolo" non dovrebbero dirsi neppure colpevoli ma soltanto sfortunati essendo loro disgraziatamente capitato di nascere $\cos ^{52}$. Clemente a sua volta si riallaccia ai criteri della pedagogia sofistica: come la virtù etica in generale non è il mero frutto di predisposizioni innate, allo stesso modo la virtù del credente non è un "privilegio di natura ${ }^{53}$ ed entrambe si conseguono solo sottoponendosi alla fatica del tirocinio educativo, all'impegno nell'apprendimento e alla perseveranza nell'esercizio $^{54}$. Così anche quando tratta problemi escatologici che a prima vista non avrebbero nulla da spartire con gli interessi di Protagora o di Socrate, il pensiero dei Padri mostra di collocarsi alla confluenza di due differenti illuminismi, ebraico e greco, accomunati dal principio della centralità dell'uomo inteso quale intelligenza operativa capace, nel bene o nel male, di costruire la propria sorte.

\section{Il dibattito sull'episodio del Faraone e sul caso di Giuda}

Non cè da meravigliarsi se la "filosofia" cristiana, nel suo duplice riferimento al moralismo farisaico e all'etica sofistico-socratico-platonica, sia assolutamente aliena tanto dalle vertiginose speculazioni di Paolo sulla grazia quanto dalla proposta sconvolgente di Giobbe che Dio stesso, se vuole, manda in frantumi il Patto e scatena sulluomo una disgrazia immeritata; per quanto - ovviamente - i relativi testi scritturali siano sovente oggetto di solenni citazioni e di dotti commentari. La grazia viene di regola intesa dai Padri in termini sinergistici, come un "soccorso" o un "premio" offerto al giusto la cui "elezione" mai prescinde dai meriti acquisiti ${ }^{55}$; il fatto che, fra l'altro, Clemente paragoni la grazia da un lato alla theia moira socratica e dall'altro alla Legge mosaica, entrambe mandate da Dio per essere di guida all'umanità,

\footnotetext{
52 Origenes, Cels. Iv, 3; In Joh. xx, 28.254.

53 Clemens Alexandrinus, Protr. 83-84; Clemens Alexandrinus, Strom. II, 3.11; viI, 7.48. Cfr. Protagoras, Diels-Kranz, fr. 22 B 3.

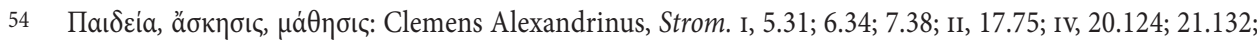
v, 1.11; VI, 11.95; 12.96; 15.121; VII, 3.9.

55 Origenes, Cels. v, 25-32; Clemens Alexandrinus, Strom. vi, 17.157; viI, 7.48.
} 
mette in luce più che mai la differenza abissale che lo separa da Paolo ${ }^{56}$. Ma se la grazia per i santi non è predestinata dapprincipio, bensì risponde alle buone azioni chessi hanno autonomamente compiuto, altrettanto si deve dire allinverso della rovina cui vanno incontro i reprobi, per esempio i personaggi negativi della Bibbia (il Faraone dell'Esodo, Giuda Iscariota) che gli gnostici consideravano o come vittime di un Dio malvagio o come dannati per natura.

Qui davvero l'esegesi dei Padri è di un'ingenuità - o di una malafede - che fa sorridere, se non si trattasse di un problema serio. Il perno dell'argomentazione si trova per la prima volta in Ireneo, che probabilmente già lo desumeva da qualcun altro: 1" indurimento del cuore" del Faraone sarebbe stato non già la causa - posta da Dio - dei suoi comportamenti ma la punizione, quindi la conseguenza, della sua incredulità. Anche Clemente conosce questa interpretazione, aggiungendo l'inedito particolare che il Faraone sarebbe stato indurito a causa del suo rifiuto di una proposta di "conversione" fattagli da Mosè. Naturalmente questo voleva dire una totale incomprensione del testo biblico, dal quale trapela con chiarezza non solo che Mosé non era motivato da alcun intento "missionario", volendo egli soltanto strappare un permesso di espatrio, ma soprattutto che l'intervento divino contro il sovrano non era affatto scaturito da una valutazione previa delle sue qualità morali. Per parare un'obiezione di questo tipo entrambi gli interpreti cristiani (o la loro fonte) spiegano l'“indurimento del cuore" come punizione anticipata di un'incredulità che, certo, nel decorso dei fatti si manifesta dopo, ma che Dio nella sua prescienza già sapeva essere una tendenza latente del malvagio Faraone ${ }^{57}$. Allora è vero che il Faraone era predestinato a comportarsi in quel modo? Assolutamente no, perché (come aveva già precisato Giustino) la prescienza divina si limita a contemplare in anticipo, senza determinarle, le azioni dell'uomo e la sanzione loro spettante, se egli liberamente sceglierà di compierle ${ }^{58}$.

Linterpretazione origeniana è più sottile, ma non meno pregiudiziale. Nel suo saggio Sulla preghiera Origene parla dell'"indurimento del cuore" alla luce della sua tesi della "pedagogia" divina che non solo mediante la rivelazione e la provvidenza della storia, ma anche agendo nei confronti dei singoli con mezzi punitivi vuol condurre l'uomo al riconoscimento della verità ${ }^{59}$. Dio dunque indurisce il Faraone non semplicemente perché lo vuole rovinare, ma per indurlo con ripetuti castighi a pentirsi:

Il motivo per cui viene indurito il cuore del Faraone non può essere quello di impedirgli, a causa dell'indurimento, di dichiarare "Il Signore è giusto, mentre io e il mio popolo siamo

\footnotetext{
56 Clemens Alexandrinus, Strom. v, 13.83; Paed. I, 68 e Strom. vi, 95-96.

57 Irenaeus, Haer. Iv, 29; Clemens Alexandrinus, Paed. I, 76.

58 Justinus, 1 Apol. 43; cfr. Clemens Alexandrinus, Strom. vi, 17.156-157; Origenes, Hom.Jer. xviII, 6; Philocalia 23.8 e 25.2. Sono le premesse medioplatoniche (Alcinous, Didascalicus 26; Hippolytus, Refut. I, 19.19; ps. Plutarchus, De fato 4-5) della scientia media teorizzata dal gesuita L. de Molina (1535-1600). 
empi" <Es., 9.27>: giacché, al contrario, proprio queste furono le sue parole. Piuttosto, egli andava ripetutamente indurito e sottoposto a sofferenze affinché non sottovalutasse l'indurimento, qualora fosse durato troppo poco, reputandolo una disgrazia [occasionale]: per questo richiedeva di subirlo più volte ancora ${ }^{60}$.

Più ampia la discussione nel grande trattato Sui principi dove confuta la tesi valentiniana secondo cui l'indurimento del Faraone stava a significare la sua natura di "ilico", quindi sprovvisto del libero arbitrio in dotazione solo agli "psichici". Ora, se la sua natura era talmente corrotta chegli mai e poi mai avrebbe obbedito al comando divino, che bisogno c'era di indurire vieppiù un cuore già sufficientemente duro di per sé? L'indurimento ha senso quando è eseguito per bloccare una possibilità di obbedienza che altrimenti sarebbe stata a disposizione del soggetto, il quale dunque era libero. D’altra parte, se i valentiniani vedono nel Dio biblico il loro "Demiurgo" che quanto meno è "giusto", come può considerarsi un atto di giustizia il mettere forzatamente un uomo nella condizione di peccare ${ }^{61}$ Invece l'unico vero Dio è sia buono sia giusto, e rivolge la medesima azione provvidenziale a tutti, santi o peccatori, come la pioggia fa crescere i frutti e le spine, senza determinare la loro bontà o malvagità ma solo permettendo il libero sviluppo della natura propria ad ogni essere. Ma non erano appunto i valentiniani a parlare della "natura"? Certo, però Origene la intende in tutt'altro modo: la natura del soggetto non è predefinita una volta per tutte bensì dipende da lui costruirla e orientarla verso il bene o verso il male accettando la correzione divina oppure cedendo a un impulso maligno. Anche qui l'indurimento va inteso come effetto e non come causa, perché «Dio non si prefigge di per sé lo scopo di indurire una persona: il suo intento infatti è buono. L'indurimento semmai si verifica in conseguenza d'un principio malvagio intrinseco alla persona stessa» ${ }^{62}$. Tuttavia la natura umana è mutevole, a seconda della maggiore o minore adesione del soggetto alle sue maligne tendenze e pertanto anche l'ostinazione che consegue dal cattivo controllo di sé non è tanto rigida da non manifestare una potenziale fragilità: difatti «lo stesso cuore indurito del Faraone talvolta si ammorbidisce e pian piano cede di fronte ai segni, dando prova che essi hanno avuto effetto su di lui: solo che, alla fine, ciò non è stato ancora sufficiente» ${ }^{63}$. Insomma,

\footnotetext{
60 Origenes, Or. 19; ib. 2: il Faraone più volte raccomanda a Mosè di "pregare" Dio per lui.

61 Origenes, De princ. III, 1.8-9. Sulla giustizia del Demiurgo cfr. Tolomeo in Epiph. 33.7; Origene si rivolge anche contro Marcione sulla cui concezione negativa del libero arbitrio v. E. Norelli, Marcione e gli gnostici sul libero arbitrio, in Perrone, Il cuore indurito.

62 Origenes, De princ. III, 1.10; cfr. Philocal. 27.

63 Origenes, De princ. III, 1.11. Anche il Commento a Romani spiega che l'indurimento è causato dall'uomo e non da Dio: «non enim quem vult deus indurat sed qui patientiae obtemperare noluerit, induratur», viI, 16 (PG 14, 1147a). L'interpretazione origeniana è ripresa da Eznik di Kolb, Su Dio 237-241; Titus Bostrensis, Contra manichaeos I, 33 (PG 18, 1124b).
} 
è mancato solo un soffio alla "conversione" del Faraone! Neppure l'ottimismo di Clemente sarebbe arrivato a un così clamoroso stravolgimento del testo biblico.

Nei Principi non si fa cenno a una prescienza divina del comportamento del Faraone perché, come sè visto, la durezza di cuore ha la sua vera causa in lui, non in Dio. Il tema compare invece in un'ulteriore disamina della questione nel Contro Celso, stavolta riguardo a Giuda. Celso accusava Gesù di aver «complottato contro un suo commensale» perché preannunciando, in quanto Dio, l'inevitabilità del tradimento di Giuda era in fondo lui stesso a render traditore ed empio quest'uomo che sedeva alla sua tavola. La risposta è che la prescienza non causa gli avvenimenti futuri da essa annunciati, i quali restano sempre passibili di accadere o non accadere ad opera degli agenti reali; quindi Giuda avrebbe potuto benissimo non tradire Gesù, allo stesso modo in cui Laio avrebbe potuto benissimo non generare Edipo evitando le conseguenze predette dalloracolo. Se prima Origene si era in parte appoggiato alla concezione stoica della "natura propria" che è plastica e non immutabile, qui sfrutta la tesi medioplatonico-peripatetica che Dio conosce in anticipo gli eventi indeterminati per quel che sono, cioè come indeterminati e non come necessari ${ }^{64}$. Ma allora perché Gesù preannuncia l'imminente tradimento in termini perentori? Il discorso origeniano sembra inclinare a una soluzione simile a quella dei medioplatonici sul caso appunto di Laio: ciò che Gesù conosceva con assoluta certezza non era tanto il verificarsi del tradimento in sé quanto l'«indole maligna» (mochthēròn èthos) di Giuda, da cui egli poteva legittimamente concludere l'assai elevata probabilità che il tradimento si verificasse, pur essendo esso un atto libero ${ }^{65}$. Io trovo questo caso estremamente illuminante per cogliere la complessità del rapporto che lega la sedicente "filosofia" cristiana alle sue fonti filosofiche greche. Origene, a un primo livello, fa uso strumentale della "filosofia della religione" medioplatonica, in cui si trattava degli oracoli pagani, per "dimostrare" contro gli gnostici la dottrina della Chiesa sul libero arbitrio; a un secondo livello però tale convergenza fra cristianesimo e platonismo ha la sua ragion d'essere non nell'esigenza di spiegare meglio la Rivelazione, data la palese incongruenza della teoria origeniana con la lettera e lo spirito del passo evangelico (anche a prescindere dall'ulteriore problema della congruenza del passo medesimo con la visione evangelica in generale), bensì nel fatto che Origene presuppone da parte sua lo stesso postulato dei medioplatonici, di Platone, e prima ancora di Protagora, di Solone, del poeta dell'“Apologia di Zeus" (dell'inizio dell'Odissea), secondo cui gli eventi dell'esistenza umana devono trovare fondamentalmente una spiegazione a partire dall'uomo stesso, dalla sua iniziativa e dal suo agire. Giac-

64 Maximus Tyrius, Dissertationes v, 2 e xiII, 5; Alexander Aphrodisiensis, De fato 30. Già Carneades (Mette, fr. 10) diceva che neppure Apollo poteva prevedere le libere azioni di Edipo.

65 Origenes, Cels. II, 20 = Philoc. 23.12-13; cfr. In ep. Rom. IV, 3; viI, 17; Philoc. 25.1-3. Stessa spiegazione in Maximus Tyrius, Dissertationes XIII, 5 e Chalcidius, In Timaeum 153-154. 
ché solo in questo modo il pensiero può svolgere un ruolo coerente con i bisogni della comunità, sociale o religiosa che sia.

\section{L'ontologia aristotelica nella confutazione del manicheismo}

Dal IV al VI sec. l'attività degli eresiologi entrò in una seconda fase avendo come obiettivo polemico il manicheismo ${ }^{66}$. La nuova religione fondata da Mani (216-277) costituiva un avversario quanto mai pericoloso perché non era una semplice setta bensì un credo con ambizioni universalistiche, con un sistema dogmatico al contempo rigido nelle sue strutture portanti e abbastanza duttile da presentarsi quale sintesi definitiva delle principali tradizioni religiose dell'Oriente (apocalittica, cristianesimo, zoroastrismo, buddhismo), e con una notevole capacità di penetrazione missionaria che le consentì di estendersi in breve tempo nel territorio dei due grandi imperi dell'epoca, romano e persiano, dal Nordafrica fino all'Asia centrale. Pur sapendosi adattare a contesti culturali e religiosi diversissimi, la dottrina manichea aveva una sua identità precisa, in cui l'elemento gnostico-cristiano è senza dubbio prevalente. L'idea centrale è sempre quella di un "incidente" primordiale in seguito al quale particelle di sostanza divina sono andate disperse nella dimensione inferiore e abbisognano di essere redente; autore di questa redenzione è Cristo, il quale è insieme il Salvatore disceso nel mondo e il salvato in quanto ciò chegli viene a recuperare non è che parte di lui stesso, o in altre parole la salvezza è il processo a due facce in cui da un lato Dio «raccoglie le sue membra» e si reintegra, dall'altro l'uomo «conosce la sua anima» e così ritrova il proprio Sé eterno ${ }^{67}$. Tuttavia a differenza dello gnosticismo che nella maggior parte dei casi è monistico e considera il male come il derivato di una degenerazione della divinità, il manicheismo è dualistico e per esso il male esisteva già da sempre come realtà indipendente, altrimenti Dio dovrebbe esserne stato la causa. Di qui la sua definizione di «dottrina delle due nature», quella divina e quella diabolica, la Luce e le Tenebre, e la descrizione della loro lotta attraverso la storia del mondo che si svolge in «tre tempi»: il primo è quello della situazione originaria in cui le due dimensioni opposte coesistono dalleternità, ma separate da una specie di cortina; nel secondo le forze delle tenebre si accorgono accidentalmente della presenza della Luce al di là della cortina e decidono di impadronirsene catturando, anzi "ingerendo" parte della sostanza luminosa divina; allora il mondo divino passa alla controffensiva, vince gli "arconti" diabolici e costruisce l'attuale mondo

\footnotetext{
66 Per una presentazione d'insieme e raccolta di documenti originali rimando al vol. da me curato Il manicheismo. Antologia dei testi (Magris, Il manicheismo). Il mio saggio Il manicheismo al tempo di Agostino (2015) è disponibile in www.researchgate.net.

67 Fr. M 21, tr. it. Magris, Il manicheismo, 159; Salmi di Tommaso 13, tr. it. ib., 281.
} 
fisico come una specie di laboratorio, dotato di meccanismi per "filtrare" la sostanza luminosa prigioniera (le anime) dalla grana materiale in cui era stata incorporata; il terzo vede il lavoro missionario e lo sforzo ascetico della Chiesa manichea, la quale annunciando il messaggio di salvezza del «Gesù di Luce» e dei suoi precursori (tra i quali Enoc, Zarathustra e Buddha) consente la liberazione di un numero crescente di anime e quindi il progressivo ritorno della sostanza di luce al proprio mondo, svuotando dallinterno il regno dei malvagi arconti. Alla fine del mondo il regno della Luce verrà reintegrato nella sua condizione originaria, mentre quello delle tenebre sarà reso per sempre inoffensivo.

Avendo trascorso entro la setta giudeocristiana di Elcaseo i primi vent'anni della sua vita, Mani si era familiarizzato con il linguaggio della tradizione apocalittica e perciò è normale che la sua dottrina ne riprenda alcuni luoghi comuni: la prescienza che Dio ha dell'intero decorso dei tre tempi ${ }^{68}$, la preesistenza celeste dei membri della Chiesa, dei quali le «forme» eterne (cioè i rispettivi «Gemelli») pregano e intercedono per loro nel mentre essi vivono temporaneamente nel mondo ${ }^{69}$. Tuttavia, come in molte forme di gnosticismo giudeocristiano, una rigorosa idea di predestinazione è assente, anzi contraria alla dottrina. Tutta la sostanza divina divorata dagli arconti è "predestinata" a «tornare al proprio luogo» $»^{70}$, senza che nessuno abbia stabilito sorti differenziate per singoli individui. Certo, alla fine accadrà che una piccola parte ne vada perduta per esser rimasta troppo attaccata alla materia: ciò è fisiologico in un processo di così vasta portata, al punto che Dio stesso lo sa e lascia fare; si tratta però delle anime che hanno volontariamente dubitato della possibilità di redimersi e pertanto hanno condiviso la sorte delle tenebre ${ }^{71}$. Vi è infatti un certo automatismo del processo di recupero della Luce per quanto riguarda la natura, ma nell'uomo la salvezza dipende unicamente dall'impegno morale della persona, dalla sua disponibilità a convertirsi, dalla sua forza nel tenere sotto controllo le «Porte dei sensi» reprimendo l'accesso di seduzioni malvagie, dalla sua perseveranza ad osservare i «precetti» della religione manichea e ad accumulare «opere» meritorie $^{72}$. Neppure l'eletto è per principio sottratto alla tentazione e alla possibilità

Fr. M 10, tr. it. Magris, Il manicheismo, 147; Compendio delle dottrine di Mani, il Buddha di luce, in Tajadod, Mani, 63.

69 Copt. Morfē: Capitoli 90, tr. it. Magris, Il manicheismo, 284.

70 Compendio, Tajadod, Mani, 65.

71 Fr. M 2, Magris, Il manicheismo, 207: «Quellenergia luminosa che a tal punto è mescolata con la tenebra da non poter esserne nuovamente divisa non è di natura uguale a quella $<$ che si salva $>[. .]<$. Il salvato infatti $>$ non pensa: Per me la mescolanza originaria con l'oscurità produce una corruzione e un peso tali che io non posso più liberarmi e separarmi dalla tenebra». Il passo mostra che la differenza di "natura" fra anima salvata e anima perduta non è oggettiva (entrambe sono sostanza luminosa) ma riflette l'atteggiamento soggettivo di fede o di disperazione: chi non vuol credere perde la sua prima natura e ne acquista un'altra peggiore.

72 Sorveglianza delle Porte: fr. M 2, tr. it. Magris, Il manicheismo, 130; Capitoli 56, tr. it. ib., 187-188. Precetti

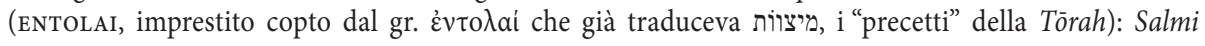


di perdere la fede ricadendo nel peccato, se non fa costantemente attenzione a trasformare l'«Uomo vecchio» che è in lui in «Uomo nuovo»" ${ }^{73}$. Nonostante l'attaccamento di Mani a Paolo, il manicheismo è non meno del cristianesimo patristico una religione della "Legge", non della "grazia". La salvezza è un risultato della libera scelta umana, non della predestinazione divina.

Poiché dunque non si poteva accusare il manicheismo di negazione fatalistica del libero arbitrio, gli eresiologi cristiani aggiustarono il tiro della loro polemica mirando al punto più problematico del sistema: il postulato dualistico per cui Dio e il male sono due "nature" originarie e indipendenti. Qui però si poneva un dilemma. Se il male esiste di per sé, la sua realtà delimita quella divina e in conseguenza Dio non è il principio assoluto di tutte le cose; ma se Dio è l'unico principio - opzione obbligata per un cristiano - bisogna considerarlo responsabile dellesistenza del male in quanto anchessa è derivata da lui. Per sbrigarsela, i Padri dovettero sfruttare fino in fondo gli espedienti della "teodicea" platonica che a sua volta era la logica conseguenza di una teologia di tipo illuministico ${ }^{74}$ : Dio è un'intelligenza operativa autrice del prodotto migliore possibile a certe date condizioni; il male è un fenomeno soltanto soggettivo perché in realtà è funzionale all'insieme e perciò a sua volta a fin di bene; l'unico male effettivo è quello prodotto dall'iniziativa umana che ne è responsabile a causa delle sue scelte sbagliate. Si delinea così l'impostazione del problema che da allora fino ad oggi è rimasta canonica nel pensiero cristiano.

Lo schema delle argomentazioni, in larghissima parte copiate dalle fonti filosofiche greche, è un lavoro compilatorio che si ripete monotono e scontato in tutti gli scrittori lasciando un'impressione di tale superficialità da dover concludere, almeno sul punto ora in questione, che questa "filosofia" patristica è un vero trionfo della retorica. Riassumiamo brevemente i contenuti. Premesso che in generale il creato non può non denotare una qualche imperfezione, perché altrimenti sarebbe stato perfetto come il Creatore e identico a lui, non ontologicamente diverso ${ }^{75}$, i Padri ribadiscono che tale intrinseca negatività è ben poca cosa di fronte alla constatazione della regolarità, della bellezza e della funzionalità del mondo naturale ${ }^{76}$. D’altronde anche gli

del Bema 239, tr. it. ib., 267; Salmi degli erranti 31, tr. it. ib., 271. Le "opere": Salmi degli erranti 30, tr. it. ib., 270; Salmi di Eraclide 5, tr. it. ib., 327; Trattato Chavannes-Pelliot, tr. it. ib., 243.

73 Cfr. Capitoli 38, Trattato sull'Intelletto di luce e Trattato Chavannes-Pelliot, tr. it. Magris, Il manicheismo, 218-247 (la metafora è deuteropaolina: Ef. 4.22 e Col. 3.9).

74 L’elemento illuministico è più essenziale di quello platonico perché proprio il platonismo aveva anche una minoritaria tendenza dualistica: le dottrine non scritte di Platone ponevano due archái, l'Uno (causa del bene) e la Diade (causa del male); alcuni medioplatonici teorizzavano due Anime del mondo, una buona e l'altra cattiva, segnatamente Plutarco, Attico e Numenio.

75 Irenaeus, Haer. Iv, 38.1; Tertullianus, Contra Marcionem II, 9.

76 Finalità e utilità degli elementi naturali: Basilius Caesariensis, Hom. in Gen. III, 5; Gregorius Nyssenus, Oratio cathec 5 (GNO Online 16); Titus Bostrensis, Contra manichaeos II, 29-32 e 36-37; Johannes Chrisostomus, De providentia 4.5 e 7.2; Theodoretus, De providentia 1-6. Utilità di bestie feroci, veleni dei serpenti ecc.: Basilius Caesariensis, Hom. in Gen. v, 3-4; IX, 6; Titus Bostrensis, Contra manichaeos II, 21-24; Eznik di Kolb, Su Dio 67-68 (già Filone d.A., S. provv. 103-105). Corpo umano: Basilius 
aspetti della natura e della vita che a noi paiono negativi non sono veramente un male $^{77}$. Già Origene aveva insistito su questo punto, riecheggiando note tematiche stoiche: le avversità che si incontrano nell'esistenza sono una "prova", un "esercizio" per la fortezza d'animo, una "scuola", una "medicina" amara ma salutare ${ }^{78}$, e sotto questo profilo anche il presunto male è una componente necessaria della "pedagogia" divina ${ }^{79}$. Con altrettanta frequenza rintrona la cantilena socratica ${ }^{80}$ che i cosiddetti beni esteriori sono irrilevanti per il cristiano e non va dunque considerato un male la loro mancanza, al contrario: la povertà insegna ad essere sobri, i torti è meglio subirli ingiustamente che farli (come affermava Socrate nel Gorgia), la malattia rende più umili, la morte ricongiunge il credente al cielo e persino se giunge prematura è un segno della misericordia divina ${ }^{81}$. A ciò si aggiunge il tema dell'ingiustizia sociale, nuovo rispetto alla trattatistica greca, su cui erano stati gli gnostici e i manichei a porre l'accento come dimostrazione che la provvidenza in questo mondo non esiste $^{82}$. Si capisce che i Padri, nel momento in cui il cristianesimo era stato appena riconosciuto come religione lecita (313) e poi come unico culto di Stato (392), impiegarono tutte le loro risorse per scansare ogni sospetto di sovversione e giustificare l'assetto esistente della società. Gli argomenti sono di tenore molto paternalistico. La distinzione di classe, la disparità fra ricchi e poveri, la divisione del lavoro, la gerarchia sociale, la severità delle leggi sono indispensabili al funzionamento dello Stato ed il cristiano deve mostrare sopportazione e ubbidienza, convinto che comunque il benessere materiale di per sé non conta e che per mal che vada ci sarà

Caesariensis, Hom. in Gen. XI, 15-17; Theodoretus, De providentia 3. Superiorità dell'uomo grazie a nous e technē: Basilius Caesariensis, Hom. in Gen. x, 6; Gregorius Nyssenus, De opificio hominis 7 (PG 44); Johannes Chrisostomus, De providentia 7.20.

77 Gregorius Nyssenus, Oratio cathechetica 8 (GNO Online 16).

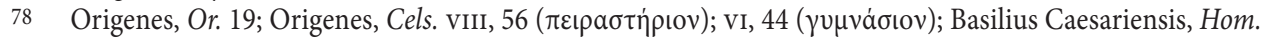

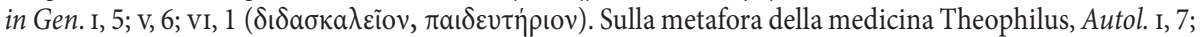
Clemens Alexandrinus, Protr., 8, Paed. I, 1; Origenes, Hom. Exod. viII, 5-6; Hom. Jer. vi, 2; xx, 3; Hom. Ezech. I, 2; Titus Bostrensis, Contra manichaeos II, 14-16; ps. Clemens Romanus, Recognitiones vII, 11; Gregorius Nyssenus, Oratio cathechetica 8 (GNO Online 16). Sulle premesse stoiche cfr. Seneca, De prov. 2.4 e 4.3. Cfr. Epictetus, Diatr. III, 23.30. La metafora del medico che guarisce dando medicine dolorose ha anche origini socratico-platoniche: Xenophon, Memorabilia I, 2.54; III, 3.9; Antisthenes, nr. 169 Giannantoni, Platone, Gorgia 34, 478a-e.

79 Origenes, Cels. Iv, 70; Hom. Exod., viII, 5-6; Hom. Num. Ix, 1; XIv, 2; Methodius, De libero arbitro 20 (Vaillant, 819); Titus Bostrensis, Contra manichaeos II, 38; Gregorius Nyssenus, Oratio catechetica 6 (GNO

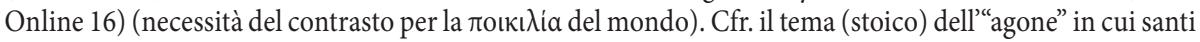
e martiri danno buona prova di sé: Titus Bostrensis, Contra manichaeos II, 9-10; Johannes Chrisostomus, De providentia 21.2 (con l'esempio di Giobbe); Ad Olympiadem 7.2; 8.13; 11.1.

80 F 158 Giannantoni = Cicero, Tusculanae v, 32.91. Lirrilevanza dei beni esteriori anche in Xenophon, Symposium, 3.8: Plato, Apologia 17, 30b; Aristophanes, Nubes 441ss., Stilpo Megaricus (fr. 15, Giannantoni, Socraticorum Reliquiae $=$ Diogenes Laërtius, Vitae II, 115). In realtà pare che Socrate avesse una condizione economica agiata secondo Aristoxenus fr. 59 (Wehrli).

81 Titus Bostrensis, Contra manichaeos II, 7-9; Johannes Chrisostomus, De providentia 16.1 (povertà, torti); Titus Bostrensis, Contra manichaeos II, 13, 16 e 23; Eznik di Kolb, Su Dio 91 (morte).

82 Sullorigine gnostica del tema delle disuguaglianze sociali cfr. Origenes, Cels., Princ. II, 9.5. 
sempre ben altra ricompensa in paradiso; eventuali ingiustizie sono colpa di chi liberamente le compie ma Dio lo permette affinché il reo si penta oppure si meriti la giusta punizione ultraterrena ${ }^{83}$.

La polemica contro il dualismo manicheo rese ancora più stretta l'adesione dei pensatori cristiani allontologia aristotelica. Sotto il profilo filosofico, infatti, la loro obiezione di maggior peso è la seguente: il male non va considerato una realtà sussistente (hypóstasis) come vorrebbero i manichei, giacché esso non è una cosa ma una "qualità" (poiótēs) delle cose, quindi un "accidente" (symbebēkós), non un'"entità" (ousía); e siccome tale qualità non potrebbe inerire a nessuna delle cose di questo mondo che sono state create buone da Dio, essa consiste soltanto in ciò che l'uomo appunto "fa" (poiêi) in quanto unico essere creato libero e capace di compiere buone o cattive azioni. Il "male" si riduce al male morale, al peccato. Ora, se la peccaminosità è un predicato attribuibile a un soggetto ovvero un accidente della sostanza "uomo" e come tale soltanto passibile di accaderle o meno, il principio (archē) del suo esserci, o non esserci, altro non è se non l'"attività pratica" (praktiké enérgeia) dell'uomo stesso, vale a dire la sua "scelta" (proáiresis), la sua "intenzione" (próthesis), il suo "raziocinio" (logismós). Dunque il male di per sé non esiste, non possiede alcuna realtà originaria, perché o si tratta di un'impressione soggettiva che l'ordine provvidenziale del mondo smentisce, oppure di una realtà effettiva che tuttavia, essendo un prodotto dell'agire umano, come tutti i prodotti realizzati da una téchnē sorge occasionalmente dal nulla per libera iniziativa di un essere intelligente ${ }^{84}$. Ma il ragionamento così limpido e persuasivo comporta una conseguenza non da poco sul piano teologico: Dio, il Creatore dell'universo, non ha creato tutto quanto avviene nell'universo perché una certa cosina - il male - ce l'abbiamo messa noi ${ }^{85}$. In fondo non cè soluzione moralistica al problema del male in ambito religioso, dal racconto biblico su Adamo ed Eva all'“Apologia di Zeus", che non implichi un'inevitabile "limitazione" della divinità dovendo essa fare spazio al potere autonomo dell'uomo di produrre qualcosa che il piano del mondo non contemplava. Ma un'idea siffatta non nasce e non può nascere dalla religione, bensì da un'istanza diversa che è la matrice dell'illuminismo: al centro è l'uomo con la sua operatività tecnica e morale

83 Theodoretus, De providentia 6-9; Johannes Chrisostomus, De providentia 9.6-7; 12.3-7; Ad Olympiadem 8 e 13 .

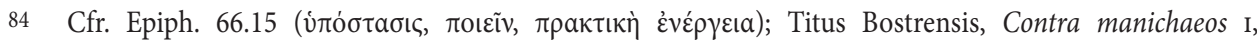

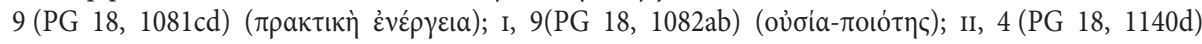

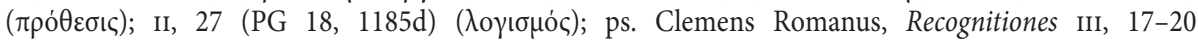
(il male è accidens e sta in hominum arbitrio); IV, 23; vIII, 51; Methodius, De libero arbitro 7 (Vaillant, 757)

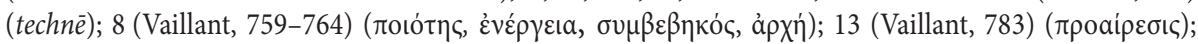
Gregorius Nyssenus, Oratio cathechetica 6 (GNO Online 16) (il male è "privazione del bene" [ảyatoũ

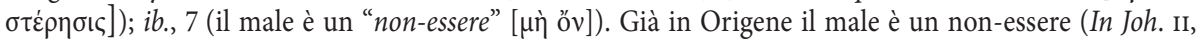

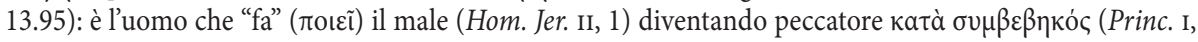
8.3; In Joh. xx, 23.199). L'essere intelligente autore del male può essere anche un angelo caduto (Satana).

Stessa tesi in Plato, Resp. II, 18, 379bc. 
(e la conseguente imputabilità sociale); di fronte a lui ciò chegli non controlla e in parte subisce, la natura, la necessità o Dio; accanto a loro eventualmente un medio, uno spazio vuoto in cui possono interagire, il caso. A questa ontologia intrinseca al pensare illuministico fondato sull'uomo la filosofia greca diede espressione con la pluralità dei fattori causali di tutto quanto avviene (cioè la necessità della natura, l'iniziativa umana e il caso o fortuna) teorizzata in età sofistica ${ }^{86}$, ripresa da Platone $e^{87}$, da Aristotele ${ }^{88}$, dai medioplatonici ${ }^{89}$. E che cosa aveva da spartire questa teoria con il cristianesimo? Forse niente. Eppure la troviamo ripetuta come cosa del tutto ovvia da esponenti della Patristica che evidentemente ne condividevano l'essenziale portata ontologica a prescindere dal fatto di formularla col linguaggio "fisico" dell'aristotelismo o con quello "teologico" del platonismo ${ }^{90}$. D’altronde il discorso filosofico della pluralità di fattori causali non affermava in sostanza il medesimo pensiero del sinergismo fra grazia e libero arbitrio che già i farisei e poi concordemente rabbini e Padri della Chiesa desumevano dalla Teologia del Patto? Accanto a Dio l'uomo ha un suo potere nel determinare il corso delle cose, poiché il realizzarsi della salvezza richiede la "collaborazione" di entrambi.

Questa massiccia adozione di concetti aristotelici da parte dei Padri senzaltro proseguiva nella direzione indicata da Clemente quando suggeriva l'impiego della logica categoriale per difendere la fede dagli eretici; ma un fenomeno curiosamente parallelo si osserva anche nel contemporaneo platonismo dove la dottrina delle categorie, già parte integrante della manualistica medioplatonica, assurge dopo Porfirio al ruolo di organon per eccellenza, lo "strumento" fondamentale per argomentare efficacemente contro filosofi di diverso orientamento e magari contro gli pseudofilosofi

86 Si veda l'interazione di anankē, technē e tychē nel trattato pseudoippocratico De arte.

87 Leges I, 4, 709b,

88 Clemens Alexandrinus, Protr. fr. 11 Ross; Aristoteles, Metaph. viI, 7, 1032a11-12; XII, 3, 1070a6; Eth. Eudem. II, 6, 1223a10-12.

89 Maximus Tyrius, Dissertationes v, 4; Chalcidius, In Timaeum 145; Diogenes Laërtius, Vitae III, 96; Ammonio in Plutarchus, Qauest.conviv. Ix, 5, 740cd; Apuleius, De Platone I, 12.

90 Per la formulazione "fisica" Basilius Caesariensis, Hom. in Gen. II, 5: il male è provocato da tò av̉

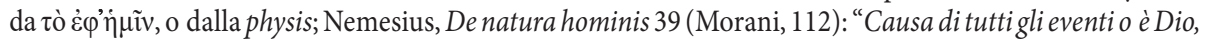

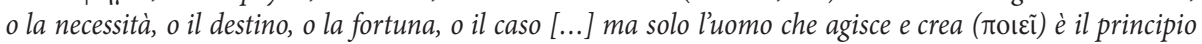

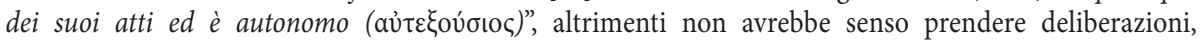
ricevere premi e punizioni ecc. La formulazione "teologica" omette il terzo fattore - il caso - lasciando solo un "condominio" di provvidenza e libero arbitrio: Nemesius, De natura hominis 40 (Morani, 116): «Essendoci ciò-che-sta-in-noi, ed essendoci anche la provvidenza, è necessario che gli eventi avvengano secondo entrambi»; Gregorius Nyssenus, Oratio cathechetica 3 (GNO Online 16): "Colui che ha il potere sull'universo, nella sua straordinaria stima per l'uomo, ha permesso che qualcosa sia pure in nostro

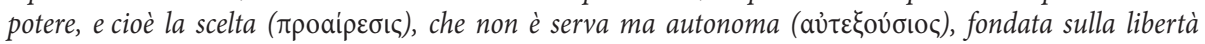

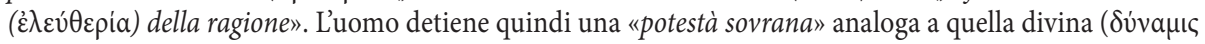

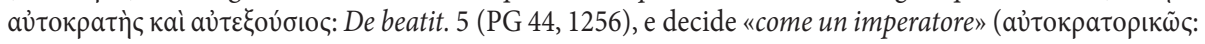
De opificio hominis (PG 44, 136bc). Si ricordi l'Intelletto "autocrate" di Anaxagoras A100 e Diels-Kranz, fr. B12. 
cristiani ${ }^{91}$. Da ciò si vede come sia semplicistico ridurre il rapporto fra cristianesimo e cultura greca al confronto di "fede" e "ragione" o al problema dell'impiego della razionalità filosofica ai fini della "Regola" ecclesiastica. La vera antitesi è trasversale, perché distingue diversi modi di intendere il cristianesimo e diversi modi di concepire la filosofia, a seconda che nell'uno o nell'altro ambito ci sia stata o meno la presa di posizione del pensiero a favore di una determinata visione dellessere, sia essa implicita od esplicita, ignara o culturalmente motivata. Certamente i Padri della Chiesa scelgono la "fede", i platonici la "ragione"; ma prima di questo è avvenuta unaltra cosa, cioè la scelta di una ontologia, che per entrambi è stata quella dell'illuminismo nella forma codificata da Aristotele.

\section{Bibliografia}

\section{Fonti}

Alexander Aphrodisiensis, De fato, trad. P. Thillet: Alexandre d'Aphrodise, Traité du Destin (a cura di P. Thillet) (Paris: Belles Lettres 1984).

Alexander Lycopolitanus, Contra Manichaeos, a cura di A. Brinkmann: Alexandri Lycopolitani Contra Manichaei opiniones disputatio (Bibliotheca Scriptorum Graecorum et Romanorum Teubneriana; Leipzig: Teubner 1889).

Aristobulus, Fragments: Fragmentsfrom Hellenistic Jewish Authors. III. Aristobulus (ed. C.R. Holladay) (Atlanta, GA: Scholars Press 1995)

von Arnim, H. (cur.), Stoicorum Veterum Fragmenta (Leipzig: Teubner 1903-1905) I-III (=SVF). Athenagoras, Ad Graecos (a cura di M. Marcovich) (Berlin: De Gruyter 1995).

Basilius Caesariensis, Homiliae in Genesim, trad. S. Giet, Basile de Cesaree, Homélies sur l' Hexaéméron, 2 ed. (a cura di S. Giet) (Paris: Cerf 1968).

Chalcidius, In Timaeum: Plao, Timaeus (a cura di J.H. Waszink) (Plato Latinus 4; London: Warburg Institute - Brill 1962).

Charlesworth, J.H. (cur.), The Old Testament Pseudoepigrapha (New York: Doubleday 1983).

Clemens Alexandrinus, Protrepticus, Paedagogus: Clemens von Alexandria, Protrepticus und Paedagogus, 3 ed. (a cura di O. Stählin) (Die griechischen christlichen Schriftsteller der ersten Jahrhunderte 12; Leipzig: Hinrichs 1972).

Clemens Alexandrinus, Stromata (a cura di O. Stählin) (Hinrichs: Leipzig 1903-1909) I-II.

91 Simplicius, In Categorias (Kalbfleisch, 14-15), mette in evidenza come la logica categoriale aristotelica sia indispensabile per la "dimostrazione". Sia i cristiani sia i platonici scartarono la dottrina stoica delle categorie (SVF II, frr. 333 e 369-404) per le sue implicazioni "materialistiche" in quanto considera come oủoía la materia (tracce di questa dottrina si trovano in Origenes, Princ. III, 6.7, dove l'oủoía è sostrato comune, non entità individuale). Il fatto è che lo stoicismo non mettendo in primo piano l'entità individuale, a differenza dell'aristotelismo, non consentiva quell'associazione fra soggetto logico e soggetto agente (onde ad es. il male diventa un "predicato" del soggetto uomo ovvero un "accidente" della sostanza uomo) così importante per la teodicea degli eresiologi. 
Clemens Romanus, Pseudo, Recognitiones. Die Pseudoklementinen. II. Rekognitionen in Rufins Übersetzung (a cura di B. Rehm) (Die griechischen christlichen Schriftsteller der ersten Jahrhunderte 51; Berlin: Akademie-Verlag 1965).

Corpus Hermeticum: Festugière, A.J. -Nock, A.D. (cur.), La révélation d'Hermès Trismégiste, 2 ed. (Paris: Les Belles Lettres 1960) $(=\mathrm{CH})$.

Diels, H. - Kranz, W. (cur.), Die Fragmente der Vorsokratiker (Berlin: De Gruyter 1966) (= Diels-Kranz).

Diodorus Tarsensis, Contra fatum: Photius, Bibliotheca (a cura di R. Henry) (Paris: Belles Lettres 1959-1967) cod. 223.

Epiphanius, Panarion: Epiphanius (a cura di K. Holl) (Leipzig: Hinrichs 1915-1922) I-II.

Epistula ad Diognetum, trad. H.I. Marrou, Anonyme, À Diognète (a cura di H.I. Marrou) (Sources chrétiennes 33 bis; Paris: Cerf 1965).

Eznik di Kolb, Su Di, otrad. L. Mariès - C. Mercier: Eznik di Kołb, De Deo (a cura di L. Mariès C. Mercier) (Turnhout: Brepols 2003).

Giannantoni, G., Socraticorum Reliquiae (Napoli: Bibliopolis 1985).

Gregorius Nyssenus, Contra Eunomium liber I (Gregorii Nysseni Opera Online 1) http://dx.doi.org/ 10.1163/2214-8728_gnoo_aGNO_1_t1 [accesso: 20.09.2021].

Gregorius Nyssenus, Contra fatum (Gregorii Nysseni Opera Online 12) http:/dx.doi.org/ 10.1163/2214-8728_gnoo_aGNO_12_t [accesso: 20.09.2021].

Gregorius Nyssenus, De mortuis (Gregorii Nysseni Opera Online 34) http://dx.doi.org/ 10.1163/2214-8728_gnoo_aGNO_34_t [accesso: 20.09.2021].

Gregorius Nyssenus, In Ecclesiasten (Gregorii Nysseni Opera Online 23) http:/dx.doi.org/ 10.1163/2214-8728_gnoo_aGNO_23_t [accesso: 20.09.2021].

Gregorius Nyssenus, Sermones (a cura di G. Heil et al.) (Gregorii Nysseni Opera 9; Brill: Leiden 1992).

Hermias, Gentilium philosophorum irrisio, trad. franc. D. Joussot: Hermias, Satire des philosophes Païens (a cura di R.P.C. Hanson) (Sources chrétiennes 388; Paris: Cerf 1993).

Hippolytus, Refutatio omnium haereseum (a cura di M. Marcovich) (Patristische Texte und Studien 25; Berlin: De Gruyter 1986).

Irenaeus, Contra haereses, trad. A. Rousseau - D. Dutreleau: Irénée de Lyon, Contre les hérésies (a cura di A. Rousseau - D. Dutreleau) (Sources chrétiennes 264; Paris: Cerf 1979) I/2.

Johannes Chrysostomus, Ad Olympiadem: Jean Chrysostome, Lettres à Olympias, 2 ed. (a cura di A.M. Malingrey) (Sources chrétiennes 13bis; Paris: Cerf 1968).

Johannes Chrysostomus, De providentia: Jean Chrysostome, Sur la Providence de Dieu (a cura di A.M. Malingrey) (Sources chrétiennes 79; Paris: Cerf 2000).

Justinus, Apologiae, trad. Justinus Martyren: Apologie pour les chrétiens (a cura di C. Munier) (Sources chrétiennes 507; Paris: Cerf 2006).

Justinus, Dialogus cum judaeo Tryphone, a cura di M. Marcovich: Iustini Martyris Dialogus cum Tryphone Patristische (Texte und Studien 47; Berlin: De Gruyter 1997).

Maximus Tyrius, Dissertationes: Maximus Tyrius dissertationes (a cura di M. Trapp) (Berlin: De Gruyter 1998).

Methodius Olympius, De libero arbitro, trad. A. Vaillant: Methodius van Olympus, Le De Autexusio de Méthode d'Olympe (a cura di A. Vaillant) (Turnhout: Brepols 1974).

Mette, J.H., "Karneades von Kyrene”, Lustrum 27 (1985) 53-141 (= Mette). 
Nag Hammadi Codices (a cura di J.M. Robinson) (Leiden: Brill 2000) (= NHC).

Nemesius Emesenus, De natura hominis (a cura di M. Morani) (Stuttgard: Teubner 1987).

Numenius, Fragmenta: Numénius, Fragments (a cura di E. Des Places) (Paris: Belles Lettres 1973) X (= Des Places).

Origen, De oratione: Origenes Werke (a cura di P. Kötschau) (Die Griechischen Christlichen Schriftsteller 3; Leipzig: Hinrichs 1899) II, 3-47.

Origen, Homiliae in Exodum, trad. M. Borret: Origène. Homélies sur l'Exode (a cura di M. Borret) (Sources chrétiennes 321; Paris: Cerf 1985).

Origenes, Contra Celsum: Origen, Gegen Celsus (a cura di P. Kötschau) (Leipzig: Hinrichs 1899) I-II.

Origenes, De principiis: Origène, Traité des Principes (a cura di H. Crouzel - M. Simonetti) (Sources chrétiennes 252, 253, 268, 269, 312; Paris: Cerf 1978-1984) I-V.

Origenes, Homiliae in Ezechielem, trad. M. Borret: Origène. Homélies sur Ézéchiel (a cura di M. Borret) (352; Paris: Cerf 1989).

Origenes, Homiliae in Genesim, 2 ed. (a cura di S. Giet) (Paris: Cerf1968).

Origenes, Homiliae in Hieremias, trad. P. Husson: Origène, Homélies sur Jérémie. I. I-XI. II. XII-XX et Homélies latines (a cura di P. Nautin) (232, 238; Paris: Cerf 1976-1977).

Origenes, Homiliae in Numeros: Origène, Homélies sur Nombres (a cura di A. Méhat) (Sources chrétiennes 415, 442; Paris: Cerf 1951).

Origenes, Homiliae in Psalmos: Origène, Homélies sur les Psaumes 36 à 38 (a cura di E. Prinzivalli) (411; Paris: Cerf 1995).

Origenes, Philocalia 21-27. Sur le libre arbitre (a cura di E. Junod) (Sources chrétiennes 226; Paris: Cerf 1976).

Simplicius, In Categorias: Commentaria in Aristotelem Graeca. VIII. Simplicii in Aristotelis Categorias commentarium (ed. K. Kalbfleisch) (Berlin: Reimer 1907).

Tatianus, Adversus Graecos; Tatiani, Oratio ad Graecos (a cura di M. Marcovich) (Berlin: De Gruyter 1995).

Tertullianus, De anima: Quinti Septimi Florentis Tertulliani, De anima (a cura di J.H. Waszink) (Amsterdam: Meulenhoff 1947).

Tertullianus, De praescriptione haereticorum; trad. D. Schleyer: Tertullian, De praescriptione haereticorum - Vom prinzipiellen Einspruch gegen die Häretiker (a cura di D. Schleyer) (Fontes Christiani 42; Turnhout: Brepols 2002).

Theodoretus, De providentia, trad. Y. Azéma: Théodoret de Cyr, Discours sur la providence (a cura di Y. Azéma) (Paris: Belles Lettres 1954).

Theophilus Antiochenus, Ad Autolicum: Tatiani Oratio ad Graecos. Theophili Antiocheni Ad Autolycum (a cura di M. Marcovich) (Patristische Texte und Studien 43-44; Berlin: De Gruyter 1995).

Theophrastus Eresius, De pietate, trad. W. Pötscher: Theophrastos, Peri eusebeias. Griechischer Text (a cura di W. Pötscher) (Leiden: Brill 1966).

Titus Bostrensis, Contra manichaeos: Patrologiae cursus completus. Series Graeca (a cura di J.P. Migne) (Paris: Migne 1857) XVIII, 1069-1264.

Usener, H., Epicurea (Lipsiae: Teubner 1887, Stuttgard: Teubner 1966) (= Usener).

Wehrli, F. (cur.), Die Schule des Aristoteles, 2 ed. (Basel: Schwabe 1967) (= Wehrli).

Wünsche, A. (cur.), Bibliotheca Rabbinica (Hildesheim: Wiss. Buchges. 1967) I-V (= Wünsche). 


\section{ALDO MAGRIS}

\section{Riferimenti bibliografici}

Desalvo, C., L'"oltre" nel presente (Milano: Vita e pensiero 1996).

von Harnack, A., Das Wesen des Christentums (Leipzig: Hinrichs 1901).

von Harnack, A., Lehrbuch der Dogmengeschichte. I. Die Entstehung des kirchlichen Dogmas

(Freiburg im Breisgau: Mohr 1886).

Jervell, J., Imago Dei (Göttingen: Vandenhoeck \& Ruprecht 1960).

Lilla, S., Clement of Alexandria (Oxford: Clarendon 1971).

Magris, A., "Platonismo e cristianesimo alla luce del Contro Celso", Discorsi di verità. Paganesi-

mo, giudaismo e cristianesimo a confronto nel Contro Celso di Origene. Atti del II convegno

del Gruppo Italiano di Ricerca su Origene e la Tradizione Alessandrina (a cura di Perrone)

(Roma: Institutum Patristicum Augustinianum 1998) 47-79.

Magris, A., "La filosofia greca e la formazione dell'identità cristiana", Annali di storia dellesegesi 21 (2004) 59-107.

Magris, A., Destino, provvidenza, predestinazione. Dal mondo antico al cristianesimo (Brescia:

Morcelliana 2012).

Magris, A., Il manicheismo. Antologia dei testi (Brescia: Morcelliana 2000).

Magris, A., Itinerari della filosofia e delle religioni (Brescia: Morcelliana 2017).

Nietzsche, F., Jenseits von Gut und Böse, http://www.nietzschesource.org/\#eKGWB/JGB [accesso: 20.09.2021].

Perrone, L. (cur.), Il cuore indurito del Faraone (Genova: Marietti 1992).

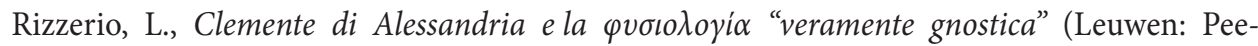
ters 1996).

Schröder, H.O., «Marionetten», Rheinisches Museum 126 (1983) 1-24.

Tajadod, N., Mani le Buddha de lumière (Paris: Cerf 1990).

Theiler, W., Zur Geschichte der teleologischen Naturbetrachtung bis auf Aristoteles (Zürich:

Füssli 1925), 2 ed. (Berlin: De Gruyter 1965). 